\title{
Oscillatory Behaviour of a First-Order Neutral Differential Equation in relation to an Old Open Problem
}

\author{
K. C. Panda, ${ }^{1}$ R. N. Rath $\mathbb{D}^{2,3}$ and S. K. Rath $^{4}$ \\ ${ }^{1}$ Department of Mathematics, Trident Academy of Technology, Bhubaneswar, Odisha, India \\ ${ }^{2}$ Department of Mathematics, VSSUT, Burla, Sambalpur 768018, Orissa, India \\ ${ }^{3}$ Khallikote Autonomous College, Berhampur 760001, Odisha, India \\ ${ }^{4}$ B.P.U.T, Rourkella, Odisha, India \\ Correspondence should be addressed to R. N. Rath; radhanathmath@yahoo.co.in \\ Received 13 February 2020; Accepted 25 March 2020; Published 1 May 2020 \\ Academic Editor: Brigitte Forster-Heinlein
}

Copyright $\odot 2020$ K. C. Panda et al. This is an open access article distributed under the Creative Commons Attribution License, which permits unrestricted use, distribution, and reproduction in any medium, provided the original work is properly cited.

In this paper, we obtain sufficient conditions for oscillation and nonoscillation of the solutions of the neutral delay differential equation $\left(y(t)-\sum_{j=1}^{k} p_{j}(t) y\left(r_{j}(t)\right)\right)^{\prime}+q(t) G(y(g(t)))-u(t) H(y(h(t)))=f(t)$, where $p_{j}$ and $r_{j}$ for each $j \quad$ and $q, u, G, H, g, h$, and $f$ are all continuous functions and $q \geq 0, u \geq 0, h(t)<t, g(t)<t$, and $r_{j}(t)<t$ for each $j$. Further, each $r_{j}(t)$, $g(t)$, and $h(t) \longrightarrow \infty$ as $t \longrightarrow \infty$. This paper improves and generalizes some known results.

\section{Introduction}

Consider the neutral delay differential equation (NDDE in short) of the first order:

$$
\begin{aligned}
& \left(y(t)-\sum_{j=1}^{k} p_{j}(t) y\left(r_{j}(t)\right)\right)^{\prime}+q(t) G(y(g(t))) \\
& -u(t) H(y(h(t)))=f(t),
\end{aligned}
$$

where $p_{j}$ and $r_{j}$ for each $j$ and $q, u, g, h, G, H$, and $f$ are in $C(\mathbb{R}, \mathbb{R})$ and $q \geq 0, u \geq 0, g(t)<t, h(t)<t$, and $r_{j}(t)<t$ for each $j$. Further, each $r_{j}(t), g(t)$, and $h(t) \longrightarrow \infty$ as $t \longrightarrow \infty$. We study the behavior of oscillation and nonoscillation of solutions of neutral differential equation (1) under the following assumptions:

$$
x G(x)>0, \quad \text { for } x \neq 0 .
$$

There exists a real-valued bounded function $F(t)$ such that

$$
F^{\prime}(t)=f(t)
$$

The function $F(t)$ in (3) satisfies

$$
\begin{gathered}
\lim _{\mathrm{t} \longrightarrow \infty} F(t)=0, \\
q(t)>0, \\
\int_{t_{0}}^{\infty} q(s) \mathrm{d} s=\infty, \\
\int_{t_{0}}^{\infty} u(t) \mathrm{d} t<\infty .
\end{gathered}
$$

$H$ is bounded and

$$
u H(u)>0, \quad \text { for } u \neq 0 .
$$

Study of the oscillatory and asymptotic behaviour of neutral delay differential equations are undertaken by numerous authors due to its various applications (see [1]) in the fields of science and technology. Results concerned with oscillation and nonoscillation of the NDDE

$$
(y(t)-p(t) y(t-\tau))^{\prime}+q(t) G(y(\sigma(t)))=f(t),
$$

are obtained in [2-5]. Further, the authors in [6-9] obtained oscillation results for the NDDE: 


$$
\begin{aligned}
& (y(t)-p(t) y(t-\tau))^{\prime}+q(t) G(y(t-\sigma)) \\
& -u(t) H(y(t-\alpha))=f(t) .
\end{aligned}
$$

The research papers $[2-5,7,8]$ and many others while studying nonlinear NDDEs assume the condition " $G$ is nondecreasing." It is found that the authors in $[3-5,7,8,10]$ use the lemmas [1] (Lemma 1.5.1 and 1.5.2) as the main tool to study $\operatorname{NDDEs}(8)$ or (9). If $\liminf _{t \rightarrow \infty} y(t)=0$ and $\lim _{t \rightarrow \infty}(y(t)-p(t) y(t-\tau))$ exists finitely then, these lemmas help us to evaluate $\lim _{t \rightarrow \infty} y(t)$. Hence, the lemmas could only be applied to study (8) or (9) where there is only one functional delay term under the derivative but could not be applied to study (1) because of the presence of more than one functional delay term under the derivative. Further, "the note [1] (notes 1.8, page 31) suggests to extend these lemmas meant for the function with one delay to multiple delays and apply it to the study of NDDE (1) with several delays." But, it seems difficult to extend these lemmas for the said purpose. The motivation behind this work is that no result in the literature appears to have an answer to the qualitative behaviour of solutions to the NDDE:

$$
\begin{aligned}
& \left(y(t)-\left(e^{-t}+e^{-4}\right) y(t-4)-\left(e^{-t}+e^{-5}\right) y(t-5)\right)^{\prime} \\
& \quad+\left(2 e^{t+1}+2 e^{t+2}+e^{2 t-3}\right) y^{3}(t-1) \\
& \quad=4 e^{-2 t+4}+4 e^{-2 t+5}+2 e^{-t}
\end{aligned}
$$

In this paper, we remove the condition " $G$ is nondecreasing" that is assumed in $[2-5,7,8]$ and study the oscillatory behaviour of solutions of the NDDE (1) and then apply the results to study the NDDE:

$$
\left(y(t)-\sum_{j=1}^{k} p_{j}(t) y\left(r_{j}(t)\right)\right)^{\prime}+v(t) G(y(g(t)))=f(t),
$$

where $v(t)$ changes sign. The oscillatory behaviour of solutions of the discrete analogue of (1) with $u=0$ is obtained in [11] by using two lemmas which are the discrete versions of Lemmas 1 and 2. We followed the methods of Rath et al. in some of our results and applied Lemmas 1 and 2 to answer the open problem [1] (Problem 2.8.3, p. 57). The oscillatory behaviour of the solution of the NDDE (1) in some results is proved to be different from that in [11].

"Let $t_{1}$ be a fixed positive real number, and

$$
t_{0}=\min \left\{\inf _{t \geq t_{1}}\left(r_{1}(t), r_{2}(t), \ldots, r_{k}(t)\right), \inf _{t \geq t_{1}} g(t), \inf _{t \geq t_{1}} h(t)\right\} \text {. }
$$

By a solution of (1), we mean a function $y \in$ $C\left(\left[t_{0}, \infty\right), \mathbb{R}\right)$ such that $y(t)-\sum_{i=1}^{k} p_{i}(t) y\left(r_{i}(t)\right)$ is differentiable on $\left[t_{0}, \infty\right)$ and the neutral equation (1) is satisfied by $y(t)$ for all $t \geq t_{1}$. It is known that (1) has a unique solution provided that an initial function $\phi \in C\left(\left[t_{0}, t_{1}\right], \mathbb{R}\right)$ is given to satisfy $y(t)=\phi(t)$ for all $t \in\left[t_{0}, t_{1}\right]$. Such a solution is said to be nonoscillatory if it is eventually positive or eventually negative, otherwise it is called oscillatory."
In this work, we assume the existence of solutions of (1) and study only their qualitative behaviour. In the sequel, unless otherwise specified, when we write a functional inequality, it will be assumed to hold for all sufficiently large values of $t$.

\section{Oscillation Results}

In this section, we present some results which prove that (5) is sufficient for any solution of (1) to be oscillatory or tending to zero as $t \longrightarrow \infty$. We need the following lemmas for our work.

Lemma 1 (see [12]). "Let $u(t)$ and $v(t)$ be two real-valued continuous functions defined for $t \geq t_{0} \geq 0$. Then,

$$
\begin{aligned}
& \liminf _{t \rightarrow \infty} u(t)+\liminf _{t \rightarrow \infty} v(t) \\
& \leq \liminf _{t \rightarrow \infty}(u(t)+v(t)) \\
& \leq \limsup _{t \rightarrow \infty} u(t)+\liminf _{t \rightarrow \infty} v(t)\left(\text { or } \liminf _{t \rightarrow \infty} u(t)\right. \\
& \left.\quad+\limsup _{t \longrightarrow \infty} v(t)\right) \\
& \leq \limsup _{t \longrightarrow \infty}(u(t)+v(t)) \\
& \leq \limsup _{t \longrightarrow \infty} u(t)+\limsup _{t \longrightarrow \infty} v(t),
\end{aligned}
$$

provided that no sum is of the form $\infty-\infty$."

Lemma 2 (see [12]). "Let $u(t)$ and $v(t)$ be two nonnegative real-valued continuous functions defined for $t \geq t_{0}$. Then,

$$
\begin{aligned}
& \lim _{t \rightarrow \infty} u(t) \times \lim _{t \rightarrow \infty} \inf _{t \rightarrow \infty} v(t) \\
& \leq \lim \inf _{t \rightarrow \infty}(u(t) \times v(t)) \\
& \leq \lim \sup _{t \longrightarrow \infty} u(t) \times \lim _{t \rightarrow \infty} \inf _{\longrightarrow} v(t)\left(\text { or lim } \inf _{t \rightarrow \infty} u(t)\right. \\
& \left.\quad \times \lim \sup _{t \longrightarrow \infty} v(t)\right) \\
& \leq \lim \sup _{t \longrightarrow \infty}(u(t) \times v(t)) \\
& \leq \lim \sup _{t \longrightarrow \infty} u(t) \times \lim \sup _{t \longrightarrow \infty} v(t),
\end{aligned}
$$

provided that no product is of the form $0 \times \infty$."

Theorem 1. Suppose that (2)-(7) hold, assume that there exists a positive constant $p$ such that $p_{j}(t)$ for $j=1,2, \ldots, k$ satisfies the following condition:

$$
\begin{gathered}
p_{j}(t) \geq 0, \text { for every } \quad j=1,2, \ldots k, \\
\sum_{j=1}^{k} \lim \sup p_{j}(t)<p<1 .
\end{gathered}
$$

Then, every solution of (1) oscillates or tends to zero as $t \longrightarrow \infty$. 
Proof. Let $y(t)$ be any solution of (1) for $t \geq t_{0}$, where $t_{0}$ is a positive real number. If it oscillates, then there is nothing to prove; otherwise, it leads to two distinct possibilities, either $y(t)>0$ or $y(t)<0$ for $t \geq t_{1}>t_{0}$. Consider the first one i.e., $y(t)>0$ eventually. There exists positive real $t_{2} \geq t_{1}$ such that for $t \geq t_{2}$, we have $y(t)>0, y(g(t))>0, y(h(t))>0$, and $y\left(r_{j}(t)\right)>0$ for each $j$. Let us define for $t \geq t_{1}$ :

$$
c(t)=\int_{t}^{\infty} u(s) H(y(h(s))) \mathrm{d} s .
$$

Clearly, due to the assumptions (6) and (7), $c(t)$ is well defined and the improper integral $c\left(t_{1}\right)$ is convergent at $\infty$, say to $\alpha$. Then, $c\left(t_{1}\right)=a(t)+c(t)$, where $a(t)=$ $\int_{t_{1}}^{t} u(s) H(y(h(s))) \mathrm{d} s$. Then, $\lim _{t \rightarrow \infty} a(t)=\alpha$. Hence, $\lim _{t \rightarrow \infty} c(t)=\lim _{t \longrightarrow \infty}\left(c\left(t_{1}\right)-a(t)\right)$. Thus, it follows that

$$
c(t) \longrightarrow 0 \text {, as } t \longrightarrow \infty \text {. }
$$

Further,

$$
c^{\prime}(t)=-u(t) H(y(h(t))) .
$$

For $t \geq t_{2}$, define

$$
\begin{aligned}
& z(t)=y(t)-\sum_{j=1}^{k} p_{j}(t) y\left(r_{j}(t)\right), \\
& w(t)=z(t)+c(t)-F(t) .
\end{aligned}
$$

From (1), (19), and (20), it follows due to (2) that

$$
w^{\prime}(t)=-q(t) G(y(g(t))) \leq 0 .
$$

Then, there exists $t_{3} \geq t_{2}$ such that $w(t)$ is monotonic and is of a constant sign for $t \geq t_{3}$. For the sake of a contradiction, assume that $y(t)$ is not bounded. Then, there exists a sequence $\left\{y\left(a_{n}\right)\right\}$ such that

$$
\begin{gathered}
a_{n} \longrightarrow \infty, y\left(a_{n}\right) \longrightarrow \infty \text { as } n \longrightarrow \infty, \\
y\left(a_{n}\right)=\max \left\{y(t): t_{3} \leq t \leq a_{n}\right\} .
\end{gathered}
$$

From (4) and (17), it follows that for $\epsilon>0$, we can find a positive real $t_{4}>t_{3}$ such that, for $t \geq t_{4}$, it implies $|F(t)|<\epsilon$ and $|c(t)|<\epsilon$. Since each $r_{j}(t) \longrightarrow \infty$ as $t \longrightarrow \infty$, we may choose $n$ large enough so that $r_{j}\left(a_{n}\right) \geq t_{4}$ for each $j$. From (15) and using (19), (20), and (23), we obtain

$$
\begin{aligned}
w\left(a_{n}\right) & =y\left(a_{n}\right)-\sum_{j=1}^{k} p_{j}\left(a_{n}\right) y\left(r_{j}\left(a_{n}\right)\right)+c\left(a_{n}\right)-F\left(a_{n}\right) \\
& \geq\left(1-\sum_{j=1}^{k} p_{j}\left(a_{n}\right)\right) y\left(a_{n}\right)-2 \epsilon>(1-p) y\left(a_{n}\right)-2 \epsilon .
\end{aligned}
$$

Taking $n \longrightarrow \infty$, we find $\lim _{t \longrightarrow \infty} w(t)=\infty$, a contradiction as $w(t)$ is monotonic decreasing. Hence, $y(t)$ is bounded which implies $w(t)$ and $z(t)$ are bounded and $\lim w(t)$ exists. Further, it follows that $\liminf _{t \rightarrow \infty} y(t)$ and $\lim \sup _{t \rightarrow \infty} y(t)$ exists. We claim $\liminf _{t \rightarrow \infty} y(t)=0$. Otherwise, let $y(t) \geq \alpha>0$. Next, since $y(t)$ is bounded above, there exists $\beta>0$ such that $y(t) \leq \beta$. Hence, we have $0<\alpha \leq y(t) \leq \beta$, which will be used for bounding the $G$ term in (1) from below.

From the continuity of $G$ and the assumption (2), it follows that there exists a positive lower bound $m$ for $G$ on $[\alpha, \beta]$. Hence, there exists $t_{5}$ such that $G(y(g(t)))>m>0$ for $t>t_{5}$. Then, integrating (21) from $t=t_{5}$ to $s$, we obtain

$$
w\left(t_{5}\right)-w(s)=\int_{t_{5}}^{s} q(t) G(y(g(t))) \mathrm{d} t \geq m \int_{t_{5}}^{s} q(t) \mathrm{d} t .
$$

In the above inequality, the left hand side is bounded, while the right hand side approaches $+\infty$, as $s \longrightarrow \infty$. Thus, we have a contradiction. This yields $\liminf _{t \rightarrow \infty} y(t)=0$. From (4), monotonic nature of $w(t)$, and (20), it follows that $\lim _{t \rightarrow \infty} z(t)$ exists finitely. Let $\lim _{t \longrightarrow \infty} z(t)=\delta$.

Then, $\quad \liminf \operatorname{in}_{t \rightarrow \infty} z(t)=\lim \sup _{t \rightarrow \infty} z(t)=\delta$. Since $p_{j}(t) \geq 0, \quad z(t) \leq y(t), \quad$ and $\quad \liminf \inf _{t \rightarrow \infty} z(t) \leq$ $\lim \inf _{t \rightarrow \infty} y(t)$, it implies $\delta \leq 0$. Then, using Lemmas 1 and 2 we obtain

$$
\begin{aligned}
0 \geq \delta & =\underset{t \longrightarrow \infty}{\limsup } z(t)=\limsup _{t \longrightarrow \infty}\left(y(t)-\sum_{j=1}^{k} p_{j}(t) y\left(\mathrm{r}_{j}(t)\right)\right) \\
& \geq \underset{t \longrightarrow \infty}{\limsup } y(t)+\liminf _{\mathrm{t} \longrightarrow \infty}\left(-\sum_{j=1}^{k} p_{j}(t) y\left(\mathrm{r}_{\mathrm{j}}(t)\right)\right) \\
& \geq \underset{t \longrightarrow \infty}{\limsup } y(t)-\limsup _{\mathrm{t} \longrightarrow \infty}\left(\sum_{j=1}^{k} p_{j}(t) y\left(r_{j}(t)\right)\right) \\
& \geq \limsup _{t \longrightarrow \infty} y(t)-\sum_{j=1}^{k} \limsup _{t \longrightarrow \infty}\left(p_{j}(t) y\left(r_{j}(t)\right)\right) \\
& \geq \limsup _{t \longrightarrow \infty} y(t)-\sum_{j=1}^{k} \limsup _{t \longrightarrow \infty} p_{j}(t) \limsup _{t \longrightarrow \infty} y\left(r_{j}(t)\right) \\
& \geq \limsup _{t \longrightarrow \infty} y(t)\left(1-\sum_{j=1}^{k} \limsup _{t \longrightarrow \infty} p_{j}(t)\right) \\
& \geq \limsup _{t \longrightarrow \infty} y(t)(1-p) \geq 0 .
\end{aligned}
$$

Hence, $\lim \sup _{t \rightarrow \infty} y(t) \leq 0$, by (15), which implies that $\lim _{t \longrightarrow \infty} y(t)=0$. If $y(t)<0$ for $t>t_{1}$, then we set $x(t)=$ $-y(t)$ to obtain $x(t)>0$ and then (1) reduces to

$$
\begin{aligned}
& \left(x(t)-\sum_{j=1}^{k} p_{j}(t) x\left(r_{j}(t)\right)\right)^{\prime}+q(t) \widetilde{G}(x(g(t))) \\
& -u(t) \tilde{H}(x(h(t)))=\widetilde{f}(t),
\end{aligned}
$$

where

$$
\tilde{f}(t)=-f(t), \quad \tilde{G}(v)=-G(-v) \quad \tilde{H}(v)=-H(-v) .
$$

Further,

$$
\widetilde{F}(t)=-F(t) \text {, implies } \quad \widetilde{F}^{\prime}(t)=\widetilde{f}(t) .
$$

In view of the above facts, it can be easily verified that $\widetilde{G}$, $\widetilde{H}$, and $\widetilde{F}$ satisfy the corresponding conditions satisfied by the functions $G, H$, and $F$ in the theorem. Proceeding as in 
the proof for the case $y(t)>0$, we may complete the proof of the theorem.

Theorem 2. Suppose that (2)-(7) hold. Assume that there exists a positive constant $p$ such that the functions $p_{j}(t)$ for $j=1,2, \ldots, k$ satisfies the condition:

$$
\begin{aligned}
& p_{j}(t) \leq 0, \quad \text { for every } j=1,2, \ldots k, \\
& \sum_{j=1}^{k} \liminf p_{j}(t)>-p>-1 .
\end{aligned}
$$

Then, every solution of (1) oscillates or tends to zero as $t \longrightarrow \infty$.

Proof. Proceeding as in the proof of Theorem 1 and setting $c(t), z(t)$, and $w(t)$ as in (16), (19), and (20), respectively, we obtain (21) and further prove $y(t)$ is bounded with $\lim \inf _{t \rightarrow \infty} y(t)=0$. From (4) and that $w(t)$ is monotonic, it follows that $\lim _{t \rightarrow \infty} w(t)=\lim _{t \rightarrow \infty} z(t)=\delta \in R$. As $z(t) \geq 0$ by (30), $\delta \geq 0$. We claim $\delta=0$, and if not, then $\delta>0$, and this implies

$$
\begin{aligned}
\delta & =\liminf _{t \longrightarrow \infty} z(t)=\liminf _{t \longrightarrow \infty}\left(y(t)-\sum_{j=1}^{k} p_{j}(t) y\left(r_{j}(t)\right)\right) \\
& \leq \liminf _{t \longrightarrow \infty} y(t)+\limsup _{t \longrightarrow \infty}\left(-\sum_{j=1}^{k} p_{j}(t) y\left(r_{j}(t)\right)\right) \\
& \leq \sum_{j=1}^{k} \limsup \left(-p_{j}(t)\right) \limsup _{t \longrightarrow \infty}\left(y\left(r_{j}(t)\right)\right) \\
& =\sum_{j=1}^{k}-\liminf _{t \longrightarrow \infty}\left(p_{j}(t)\right) \limsup _{t \longrightarrow \infty}\left(y\left(r_{j}(t)\right)\right) \\
& \leq p \limsup _{t \longrightarrow \infty}(y(t)) \leq p \alpha .
\end{aligned}
$$

Hence, we get

$$
\alpha \geq \frac{\delta}{p}>\delta .
$$

Again

$$
\begin{aligned}
\delta & =\underset{t \longrightarrow \infty}{\limsup } z(t)=\limsup _{t \longrightarrow \infty}\left(y(t)-\sum_{j=1}^{k} p_{j}(t) y\left(r_{j}(t)\right)\right) \\
& \geq \limsup _{t \longrightarrow \infty} y(t)+\liminf _{t \longrightarrow \infty}\left(-\sum_{j=1}^{k} p_{j}(t) y\left(r_{j}(t)\right)\right) \\
& =\underset{t \longrightarrow \infty}{\limsup } y(t)+\liminf _{t \longrightarrow \infty}\left(\sum_{j=1}^{k}\left(-p_{j}(t)\right) y\left(r_{j}(t)\right)\right) \\
& \geq \underset{t \longrightarrow \infty}{\limsup } y(t)+\sum_{j=1}^{k} \liminf _{t \longrightarrow \infty}\left(\left(-p_{j}(t)\right) y\left(r_{j}(t)\right)\right) \\
& \geq \limsup _{t \longrightarrow \infty} y(t)+\sum_{j=1}^{k} \liminf _{t \longrightarrow \infty}\left(-p_{j}(t)\right) \liminf _{t \longrightarrow \infty} y\left(r_{j}(t)\right) \\
& =\limsup _{t \longrightarrow \infty} y(t)=\alpha,
\end{aligned}
$$

a contradiction due to the inequality (32). Hence, we conclude $\delta=0$ and from $z(t)>y(t)$, it follows that $\lim _{t \rightarrow \infty} y(t) \leq 0$. Hence, $\lim _{t \rightarrow \infty} y(t)=0$.
The proof for the case $y(t) \leq 0$ for large $t$ is similar. Hence, the theorem is proved.

Theorem 3. Suppose that (2)-(7) hold. Assume that the functions $p_{j}(t)$ for $j=1,2, \ldots, k$ satisfies the condition:

$$
\begin{aligned}
& p_{j}(t)<0, \quad \text { for every } j=1,2, \ldots k \text { and there exists, } \\
& \quad i \in\{1,2,3, \ldots, k\} \text { such that } \\
& \lim \sup p_{i}(t)-\sum_{j \neq i} \liminf p_{j}(t)<-1 .
\end{aligned}
$$

Then, every solution of (1) oscillates or tends to zero as $t \longrightarrow \infty$.

Proof. Proceeding as in the proof of Theorem 1 and setting $c(t), z(t)$, and $w(t)$ as in (16), (19), and (20), respectively, we obtain (21) and further prove $y(t)$ is bounded with $\liminf _{t \rightarrow \infty} y(t)=0$. From (4) and that $w(t)$ is monotonic, it follows that $\lim _{t \rightarrow \infty} w(t)=\lim _{t \rightarrow \infty} z(t)=\delta \in R$. As $z(t) \geq 0$, $\delta \geq 0$. We claim $\delta=0$. If not, then $\delta>0$, and this implies

$$
\begin{aligned}
\delta= & \liminf _{t \longrightarrow \infty} z(t)=\liminf _{t \longrightarrow \infty}\left(y(t)-\sum_{j=1}^{k} p_{j}(t) y\left(r_{j}(t)\right)\right) \\
\leq & \limsup _{t \longrightarrow \infty}\left(y(t)+\sum_{j \neq i}-p_{j}(t) y\left(r_{j}(t)\right)\right) \\
& +\liminf _{t \longrightarrow \infty}\left(-p_{i}(t) y\left(r_{i}(t)\right)\right) \\
\leq & \limsup _{t \longrightarrow \infty} y(t)+\limsup _{t \longrightarrow \infty} \sum_{j \neq i}-p_{j}(t) y\left(r_{j}(t)\right) \\
& +\limsup _{t \longrightarrow \infty}\left(-p_{i}(t)\right) \liminf _{t \longrightarrow \infty}\left(y\left(r_{i}(t)\right)\right) \\
\leq & \limsup _{t \longrightarrow \infty} y(t)+\sum_{j \neq i} \limsup _{t \longrightarrow \infty}\left(-p_{j}(t)\right) \limsup _{t \longrightarrow \infty}\left(y\left(r_{i}(t)\right)\right) \\
\leq & \limsup _{t \longrightarrow \infty}(y(t))\left[1-\sum_{j \neq i} \liminf _{t \longrightarrow \infty} p_{j}(t)\right] .
\end{aligned}
$$

Again, we have

$$
\begin{aligned}
\delta= & \limsup _{t \longrightarrow \infty} z(t)=\limsup _{t \longrightarrow \infty}\left(y(t)-\sum_{j=1}^{k} p_{j}(t) y\left(r_{j}(t)\right)\right) \\
\geq & \liminf _{t \longrightarrow \infty} y(t)+\limsup _{t \longrightarrow \infty}\left(-\sum_{j=1}^{k} p_{j}(t) y\left(r_{j}(t)\right)\right) \\
= & \underset{t \longrightarrow \infty}{\limsup }\left(-p_{i}(t) y\left(r_{i}(t)\right)\right)+\liminf _{t \longrightarrow \infty} \sum_{j \neq i}\left(-p_{j}(t) y\left(r_{j}(t)\right)\right) \\
\geq & \limsup _{t \longrightarrow \infty}\left(y\left(r_{i}(t)\right)\right) \liminf _{t \longrightarrow \infty}\left(-p_{i}(t)\right) \\
& +\sum_{j \neq i} \liminf _{t \longrightarrow \infty}\left(\left(-p_{j}(t)\right) y\left(r_{j}(t)\right)\right) \\
\geq & \limsup _{t \longrightarrow \infty} y(t)\left(-\limsup _{t \longrightarrow \infty} p_{i}(t)\right) \\
& +\sum_{j \neq i} \liminf _{t \longrightarrow \infty}\left(-p_{j}(t)\right) \liminf _{t \longrightarrow \infty} y\left(r_{j}(t)\right) \\
\geq & \limsup _{t \longrightarrow \infty} y(t)\left(-\limsup _{t \longrightarrow \infty} p_{i}(t)\right) .
\end{aligned}
$$


From (35) and (36), it follows that

$\limsup _{t \rightarrow \infty} y(t)\left(\left(\sum_{j \neq i} \liminf p_{j}(t)\right)-1-\lim \sup p_{i}(t)\right) \leq 0$.

Using (34), we obtain $\lim _{t \rightarrow \infty} y(t)=0$. Thus, the theorem is proved.

For the next two results we assume

$$
-b_{j} \leq p_{j}(t) \leq 0, \quad j=1,2, \ldots, k .
$$

Note that (38) is less restrictive than (34).

Theorem 4. Suppose that (2), (3), (6), and (7) hold. Assume that (38) holds. If

$$
\begin{gathered}
\liminf _{t \longrightarrow \infty} F(t)=-\infty, \\
\limsup _{t \longrightarrow \infty} F(t)=+\infty,
\end{gathered}
$$

then every solution of (1) oscillates.

Proof. Let $y(t)$ be a nonoscillatory solution of (1) for $t \geq t_{1}$. If $y(t)>0$, then set $c(t), z(t)$, and $w(t)$ as in (16), (19), and (20), respectively, to obtain (21) for $t \geq t_{2}>t_{1}$. As $y(t)>0$ and each $p_{j}(t) \leq 0$, then $z(t) \geq 0$, and by (7), we have $c(t) \geq 0$. From (21), it follows $w(t) \geq 0$ or $w(t) \leq 0$ for $t \geq t_{3}>t_{2}$. If $w(t) \leq 0$, then $(z(t)+c(t)-F(t)) \leq 0$. This implies $\quad F(t) \geq z(t)+c(t) \geq 0$. Consequently, $\liminf \operatorname{in}_{t \rightarrow \infty} F(t) \geq 0$, a contradiction. If $w(t) \geq 0$ for $t \geq t_{3}$, then $\lim _{t \longrightarrow \infty} w(t)=\alpha \in \mathbb{R}$. Clearly,

$$
z(t)+c(t)=w(t)+F(t) .
$$

We notice that

$$
\begin{aligned}
0 & \leq \liminf (z(t)+c(t)) \leq \limsup _{t \longrightarrow \infty} w(t)+\liminf _{t \longrightarrow \infty} F(t) \\
& =\lim _{t \longrightarrow \infty} w(t)+\liminf _{t \longrightarrow \infty} F(t) \\
& =-\infty,
\end{aligned}
$$

a contradiction. Hence, $y(t)<0$ for $t \geq t_{1}$. Then, again setting $c(t), z(t)$, and $w(t)$ as in (16), (19), and (20), respectively, we obtain

$$
w^{\prime}(t)=-q(t) G(y(g(t))) \geq 0, \quad \text { for } t \geq t_{2},
$$

As $y(t)<0$ and each $p_{j}(t) \leq 0$, then $z(t) \leq 0$, and by (7), we have $c(t) \leq 0$. From (42), it follows $w(t) \geq 0$ or $w(t) \leq 0$ for $t \geq t_{3}>t_{2}$. If $w(t) \geq 0$, then $(z(t)+c(t)-F(t)) \geq 0$. This implies $F(t) \leq z(t)+c(t) \leq 0$. Consequently, $\limsup \sup _{t \rightarrow \infty}$ $F(t) \leq 0$, a contradiction.

If $w(t) \leq 0$ for $t \geq t_{3}$, then $\lim _{t \rightarrow \infty} w(t)=\beta \in \mathbb{R}$. From (40), we find that

$$
\begin{aligned}
0 & \geq \limsup (z(t)+c(t)) \geq \liminf _{t \longrightarrow \infty} w(t)+\limsup _{t \longrightarrow \infty} F(t) \\
& =\lim _{t \longrightarrow \infty} w(t)+\limsup _{t \longrightarrow \infty} F(t) \\
& =\infty
\end{aligned}
$$

a contradiction. Hence, $y(t)$ is oscillatory. Thus, the proof is complete.

Note that the above theorem is independent of any assumption on $q(t)$. Thus, it holds even for conditions weaker than (5). Further, the above theorem does not hold for the homogeneous NDDE associated with (1). Next, we present the following result which holds for the homogeneous NDDE associated with (1).

Theorem 5. Assume conditions (2)-(4), (6), (7), and (38) to hold. Further, assume $g\left(r_{i}(t)\right)=r_{i}(g(t))$ for $i=1,2, \ldots, k$. Suppose that there exists a real $\alpha>0$ such that $r_{i}^{\prime}(t) \geq 1 / \alpha$. Further, suppose that there exists a real $\mu>0$ such that for $x_{i}>0, i=1,2, \ldots, k+1$ and $u>0$,

$$
\begin{aligned}
& \sum_{i=1}^{k+1} G\left(x_{i}\right) \geq \mu G\left(\sum_{i=1}^{k+1} x_{i}\right), \\
& G(u) G\left(x_{i}\right) \geq G\left(u x_{i}\right) .
\end{aligned}
$$

Let

$$
G(-u)=-G(u)
$$

$$
\begin{aligned}
& \int_{t_{1}}^{\infty} q^{*}(t)=\infty \text {, where } q^{*}(t) \\
& \quad=\min \left\{q(t), q\left(r_{1}(t)\right), q\left(r_{2}(t)\right), \ldots, q\left(r_{k}(t)\right)\right\} .
\end{aligned}
$$

Then, every solution of (1) oscillates or tends to zero as $t \longrightarrow \infty$.

Proof. Let $y(t)$ be a nonoscillatory solution of (1) for $t \geq t_{1}$. Then, $y(t)>0$ or $y(t)<0$ for $t>t_{2} \geq t_{1}$. Let $y(t)>0$ for $t>t_{2}$. Setting $c(t), z(t)$, and $w(t)$ as in (16), (19), and (20), respectively, we obtain (21). Note that (18) and (17) also hold. As $y(t)>0$ and each $p_{j}(t) \leq 0$, then $z(t) \geq 0$. From (21), (4), and (17), it follows $\lim _{t \rightarrow \infty} w(t)=\lim _{t \rightarrow \infty} z(t)=\delta \geq 0$. If $\delta=0$, then $\lim _{t \rightarrow \infty} y(t)=0$ from the fact $y(t) \leq z(t)$. For the sake of contradiction, let $\delta>0$. We choose $\epsilon>0$ such that $\delta>\epsilon$, and we have $\delta-\epsilon \leq z(t) \leq \delta+\epsilon$ for $t \geq t_{3}>t_{2}$. As $G$ is continuous and (2) holds, we have

$$
a \leq G(z(t)) \leq b \text { for } t \geq t_{4} \text {. }
$$

From (21), we have

$$
w^{\prime}(t)+q(t) G(y(g(t)))=0 .
$$

Replace $t$ by $r_{i}(t)$ in (48), then we obtain, for $i=1,2, \ldots, k$,

$$
w^{\prime}\left(r_{i}(t)\right)+q\left(r_{i}(t)\right) G\left(y\left(g\left(r_{i}(t)\right)\right)\right)=0 .
$$

On multiplying (49) by $G\left(-p_{i}(g(t))\right)$ and then adding the corresponding $k$ equations for $i=1,2, \ldots, k$ to (48), we obtain

$$
\begin{gathered}
w^{\prime}(t)+q(t) G\left(y(g(t))+\sum_{i=1}^{k} G\left(-p_{i}(g(t))\right) w^{\prime}\left(r_{i}(t)\right)\right. \\
+\sum_{i=1}^{k} G\left(-p_{i}(g(t))\right) q\left(r_{i}(t)\right) G\left(y\left(g\left(r_{i}(t)\right)\right)\right)=0 .
\end{gathered}
$$


From (38), we find $b_{j}>0$ such that $0 \leq-p_{j}(t) \leq b_{j}$ for each $j$. Then, this implies due to continuity of $G$ and (2) that there exists a real $d>0$ such that $G\left(-p_{j}(t)\right)<d$ for each $j$.
Note that $w^{\prime}<0$, and then using $r_{i}(g(t))=g\left(r_{i}(t)\right)$, (44), (46), and (47) in (50), we obtain

$$
\begin{aligned}
& 0 \geq w^{\prime}(t)+\mathrm{d} \sum_{i=1}^{k} w^{\prime}\left(r_{i}(t)\right)+q^{*}(t)\left(G(y(g(t)))+\sum_{i=1}^{k} G\left(-p_{i}(g(t))\right) G\left(y\left(g\left(r_{i}(t)\right)\right)\right)\right) \\
& \geq w^{\prime}(t)+\mathrm{d} \sum_{i=1}^{k} w^{\prime}\left(r_{i}(t)\right)+q^{*}(t)\left(G(y(g(t)))+\sum_{i=1}^{k} G\left(-p_{i}(g(t))\left(y\left(g\left(r_{i}(t)\right)\right)\right)\right.\right. \\
& \geq w^{\prime}(t)+\mathrm{d} \sum_{i=1}^{k} w^{\prime}\left(r_{i}(t)\right)+\mu q^{*}(t) G\left((y(g(t)))+\sum_{i=1}^{k}-p_{i}(g(t))\left(y\left(g\left(r_{i}(t)\right)\right)\right)\right) \\
& \geq w^{\prime}(t)+\mathrm{d} \sum_{i=1}^{k} w^{\prime}\left(r_{i}(t)\right)+\mu q^{*}(t) G\left((y(g(t)))+\sum_{i=1}^{k}-p_{i}(g(t)) y\left(g\left(r_{i}(t)\right)\right)\right) \\
& \geq w^{\prime}(t)+\mathrm{d} \alpha \sum_{i=1}^{k} w^{\prime}\left(r_{i}(t)\right) r_{i}^{\prime}(t)+\mu q^{*}(t) G(z(g(t))) \\
& \geq w^{\prime}(t)+\mathrm{d} \alpha \sum_{i=1}^{k} w^{\prime}\left(r_{i}(t)\right) r_{i}^{\prime}(t)+a \mu q^{*}(t) .
\end{aligned}
$$
obtain

Integrating the above inequality from $t_{5}\left(>t_{4}\right)$ to $s$, we

$$
w\left(t_{5}^{\prime}\right)-\frac{a \mu}{1+k d \alpha} \int_{t_{5}}^{s} q^{*}(t) \mathrm{d} t \geq w(s),
$$

where $t_{5}^{\prime}=\min \left\{r_{i}\left(t_{5}\right): i=1,2, \ldots, k\right\}$. Then, taking limit $s \longrightarrow \infty$, we obtain $w(s) \longrightarrow-\infty$, a contradiction, due to (46). Thus, $\lim _{t \rightarrow \infty} y(t)=0$. The proof for the case when $y(t)$ is eventually negative is similar.

Note that the above result holds for $f(t) \equiv 0$. Further note that condition (46) implies (5), but the converse is not necessarily true. However, if $q(t)$ is monotonic, then both the conditions are equivalent.

Next, we intend to present a result where $p_{j}(t)$, $j=1,2,3, \ldots, k$, satisfies the following condition:

$$
\begin{aligned}
& p_{j}(t)>0, \quad \text { for every } j=1,2, \ldots k \text { and there exists, } \\
& \quad i \in\{1,2,3, \ldots, k\} \text { such that }
\end{aligned}
$$

$$
\liminf p_{i}(t)-\sum_{j \neq i} \lim \sup p_{j}(t)>1 \text {. }
$$

For that purpose, we give an example which would lead us to our next result.

Example 1. Consider the first-order NDDE with variable several delay:

$$
\begin{aligned}
& \left(y(t)-\left(e^{-t}+\sqrt{e}\right) y(t-1)-\left(e^{-t}+e^{2}\right) y(t-2)\right)^{\prime}+ \\
& e y(t-3 / 2)=0 .
\end{aligned}
$$

Note that, in the above NDDE with the several delay term under a derivative sign, $p_{1}(t)=\left(e^{-t}+\sqrt{e}\right)$ and $p_{2}(t)=\left(e^{-t}+e^{2}\right)$ satisfy (53). This NDDE has an unbounded solution $y(t)=e^{t}$ tending to $\infty$ as $t \longrightarrow \infty$, unlike other results presented so far.

The above example is the motivating point to the statement of our next result.

Theorem 6. Suppose that (2)-(7) hold. Assume that the functions $p_{j}(t)$ for $j=1,2, \ldots, k$ satisfy condition (53). Then, every bounded solution of (1) oscillates or tends to zero as $t \longrightarrow \infty$.

Proof. If $y(t)$ is any positive solution of (1), then proceeding as in the proof of Theorem 3, with the application of Lemmas 1 and 2 we obtain

$$
\begin{aligned}
& \delta=\liminf _{t \longrightarrow \infty} z(t) \leq \alpha\left(1-\liminf _{t \longrightarrow \infty} p_{i}(t)\right), \\
& \delta=\limsup _{t \longrightarrow \infty} z(t) \geq-\alpha\left(\sum_{j \neq i} \limsup _{t \longrightarrow \infty} p_{j}(t)\right),
\end{aligned}
$$

where $\alpha=\lim \sup _{t \rightarrow \infty} y(t)$ and $\delta=\lim _{t \rightarrow \infty} z(t)$. From these two inequalities and (53), we obtain $\delta=\alpha=0$. This completes the proof of the theorem.

Theorem 7. Suppose that (2) and (5)-(7) hold. Let $p_{j}(t), j=1,2, \ldots, k$, satisfy (53). Consider the homogeneous NDDE:

$$
\left(y(t)-\sum_{j=1}^{k} p_{j}(t) y\left(r_{j}(t)\right)\right)^{\prime}+q(t) G(y(g(t)))=f(t) .
$$

Then, every bounded solution $y(t)$ of (56) oscillates. 
Proof. Let $y(t)$ be an eventually positive solution of (56). Then, setting $z(t)$ as in (19) we obtain

$$
z^{\prime}(t)=-q(t) G(y(g(t))) \leq 0,
$$

and it follows that $z(t)$ is monotonic and of constant sign on some interval $\left[t_{1}, \infty\right)$. By this, we have two distinct possibilities, i.e., $z(t)>0$ or $z(t)<0$. As $p_{j}(t), j=1,2, \ldots, k$, satisfies (53), then let $i=1$. Suppose that $y(t)$ is bounded. This implies $z(t)$ is bounded. As $z(t)$ is monotonic also then $\lambda:=\lim _{t \rightarrow \infty} z(t)$ exists as a finite number. If $z(t)>0$ or $z(t)<0$, then $\lim _{t \rightarrow \infty} z(t)$ exists finitely. Integrating (57) from $t=t_{0}$ to $s$ and taking limit $s \longrightarrow \infty$, we obtain

$$
\int_{t_{0}}^{\infty} q(t) G(y(g(t))) \mathrm{d} t<\infty .
$$

Now, we claim that $\lim \inf y(t)=0$. Taking integration on (57),

$$
z(t)-\lambda=\int_{t}^{\infty} q(s) G(y(g(s))) \mathrm{d} s .
$$

Since $z(t)$ is bounded, the above integral is convergent. This in turn, by (5), it implies that $\liminf _{s \rightarrow \infty}$ $G(y(g(s)))=0$. As $G(x) \neq 0$ for $x \neq 0, \quad \liminf _{s \longrightarrow \infty}$

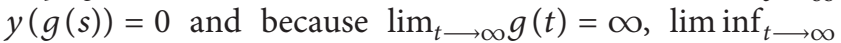
$y(t)=0$. Following the line of proof as in Theorem 6 , we find that $\lim _{t \longrightarrow \infty} z(t)=\delta=0$, which implies that $z(t)>0$ because $z$ is decreasing. Since $p_{i}(t) \geq 1$, then $y(t)>$ $\sum_{j=1}^{k} p_{j}(t) y\left(r_{j}(t)\right) \geq y\left(r_{i}(t)\right)$. Consequently, $\liminf \operatorname{int}_{t \rightarrow \infty}$ $y(t) \neq 0$, a contradiction. Hence, the bounded solution $y(t)$ cannot be eventually positive. The proof for the case when $y(t)$ is bounded and eventually negative solution of (56) is similar. Thus, every bounded solution $y(t)$ oscillates, and the theorem is proved.

Example 2. Consider the first-order NDDE (10) with several delays under the derivative. It satisfies all the conditions of Theorem 1. As such the equation has a solution $y(t)=e^{-t}$ which tends to zero as $t \longrightarrow \infty$. However, no result in the literature appears to have an answer to the qualitative behaviour of solutions to this NDDE.

Remark 1. In this section, we have not assumed the condition that $G$ is nondecreasing unlike the authors in $[3,4,10]$.

\section{Nonoscillation Results}

In this section, we show that (5) is necessary for every solution of (1) to be oscillatory or tending to zero as $t \longrightarrow \infty$. Or equivalently $\int_{t_{0}}^{\infty} q(t) \mathrm{d} t<\infty$ is sufficient for (1) to have a bounded positive solution which does not tend to zero as $t \longrightarrow \infty$, even if the limit exists. For this, we need the following lemma.

Lemma 3 (Krasnoselskii's fixed-point theorem [13]). Let $X$ be a Banach space and $S$ be a bounded closed convex subset of $X$. Let $A$ and $B$ be operators from $S$ to $X$ such that $A x+$
$B y \in S$ for every pair of $x, y \in S$. If $A$ is a contraction and $B$ is completely continuous, then the equation

$$
A x+B x=x,
$$

has a solution in $S$.

Theorem 8. Assume that (3) and (6) hold. Further, assume that one of the conditions of (15) or (30) hold. Then, (5) is a necessary condition for all solution of (1) to be oscillatory or tending to zero as $t \longrightarrow \infty$.

Proof. Suppose condition (15) holds. The proof for the case when (30) holds, would follow on similar lines. Assume for the sake of contradiction, that (5) does not hold. This implies that there exists real $t_{0}$ such that

$$
\int_{t_{0}}^{\infty} q(t) \mathrm{d} t<\infty
$$

Thus, all we need to show is the existence of a bounded solution $y(t)$ of (1) with $\liminf _{t \rightarrow \infty} y(t)>0$. From (3), we find a positive constant $c$ and a positive real $t_{1}>t_{0}>0$ such that

$$
|F(t)|<c, \text { for } t \geq t_{1}
$$

Choose a positive constant $L$ such that $L \geq 5 c / 1-p$. Since $G \in C(\mathbb{R}, \mathbb{R})$, then let

$$
\begin{aligned}
& \beta=\max \{|G(x)|: c \leq x \leq L\}, \\
& \gamma=\max \{|H(x)|: c \leq x \leq L\} .
\end{aligned}
$$

Let

$$
\mu=\max \{\beta, \gamma\} \text {. }
$$

From (6), we find $t_{2}>t_{1}$ such that $t>t_{2}$ implies

$$
\mu \int_{t}^{\infty} u(s) \mathrm{d} s<c .
$$

Then by using (61), one can fix $t_{3}>t_{2}$ such that for $t \geq t_{3}$ it follows that

$$
\mu \int_{t}^{\infty} q(s) \mathrm{d} s<c
$$

Choose $T_{1}>t_{3}$ such that

$$
T_{0}=\min \left\{g\left(T_{1}\right), h\left(T_{1}\right), r_{1}\left(T_{1}\right), r_{2}\left(T_{1}\right), \ldots, r_{k}\left(T_{1}\right)\right\}
$$

Let $X=B C\left(\left[T_{0}, \infty\right), R\right)$, a Banach space of real-bounded, continuous functions with norm $\|x\|=$ $\sup _{t \geq T_{0}}|x(t)|<\infty$. Define

$$
S=\left\{y \in X: c \leq y(t) \leq L, t \geq T_{0}\right\} .
$$

Clearly $S$ is a bounded closed and convex subset of $X$. Define two operators $A$ and $B: S \longrightarrow X$ as follows. For $y \in S$, define 


$$
\begin{aligned}
& A y(t)= \begin{cases}A y\left(T_{1}\right), & T_{0} \leq t \leq T_{1}, \\
\sum_{j=1}^{k} p_{j}(t) y\left(r_{j}(t)\right)+F(t)+3 c, & t \geq T_{1},\end{cases} \\
& B y(t)= \begin{cases}B y\left(T_{1}\right), & T_{0} \leq t \leq T_{1}, \\
\int_{t}^{\infty} q(s) G(y(g(s))) \mathrm{d} s-\int_{t}^{\infty} u(s) H(y(h(s))) \mathrm{d} s, & t \geq T_{1} .\end{cases}
\end{aligned}
$$

First, we show that if $x, y \in S$, then $A x+B y \in S$. In fact, $x=x(t)$ and $y=y(t) \in S$ and for $t \geq T_{1}$, we obtain

$$
\begin{aligned}
A x(t)+B y(t) \leq & \sum_{j=1}^{k} p_{j}(t) x\left(r_{j}(t)\right)+3 c \\
& +\int_{t}^{\infty} q(s)|G(y(g(s)))| \mathrm{d} s+|F(t)| \\
\leq & p L+5 c \leq L .
\end{aligned}
$$

On the other hand, for $t \geq T_{1}$,

$$
\begin{aligned}
A x(t)+B y(t) & \geq 3 c-\int_{t}^{\infty} u(s)|H(y(h(s)))| \mathrm{d} s-|F(t)| \\
& \geq 3 c-c-c \geq c .
\end{aligned}
$$

Hence,

$$
c \leq A x(t)+B y(t) \leq L, \text { for } t \geq T_{1} .
$$

Thus, we proved that $A x+B y \in S$ for any $x, y \in S$. Next, we show that $A$ is a contraction on $S$. In fact for $x, y \in S$ and $t \geq T_{1}$ we have

$$
\begin{aligned}
\|(A x(t)-(A y(t) \| & \leq \sup _{t \geq T_{0}} \sum_{j=1}^{k}\left|p_{j}(t)\right| \mid x\left(r_{j}(t)-y\left(r_{j}(t) \mid\right.\right. \\
& \leq \sum_{j=1}^{k} \sup _{t \geq T_{0}}\left(\left|p_{j}(t)\right|\left|x\left(r_{j}(t)-y\left(r_{j}(t)\right)\right)\right|\right. \\
& \leq \sum_{j=1}^{k}\left(\sup _{t \geq T_{0}}\left|p_{j}(t)\right|\right)\left(\sup _{t \geq T_{0}}\left|x\left(r_{j}(t)-y\left(r_{j}(t)\right)\right)\right|\right) \\
& \leq\|x(t)-y(t)\| \sum_{j=1}^{k}\left(\sup _{t \geq T_{0}}\left|p_{j}(t)\right|\right) \\
& \leq p\|x-y\| .
\end{aligned}
$$

This implies $\mathrm{A}$ is a contraction because $0<p<1$. Next, by following the arguments given in [14] (Theorem 2.2), we show that $B$ is completely continuous. Then, by Lemma 3 , we can find $x_{0}$ in $S$ such that $A x_{0}+B x_{0}=x_{0}$. Clearly, $x_{0}(t)$ is a bounded, positive solution of (1) with limit infimum greater than or equal to $c>0$. Thus, the theorem is proved.
Remark 2. Theorems for the case $p_{j}(t)$ satisfying (34) or (53) may be proved as in the proof of the above theorem with the following definition of the operators $A$ and $B$. For $x \in S$, define

$$
\begin{aligned}
& A x(t)= \begin{cases}A x\left(T_{1}\right), & \text { if } t \in\left[T_{0}, T_{1}\right], \\
\frac{x\left(r_{i}^{-1}(t)\right)}{p_{i}\left(r_{i}^{-1}(t)\right)}-\frac{\sum_{j \neq i} p_{j}\left(r_{i}^{-1}(t) x\left(r_{j}\left(r_{i}^{-1}(t)\right)\right)\right)}{p_{i}\left(r_{i}^{-1}(t)\right)}+\frac{b \lambda}{p_{i}\left(r_{i}^{-1}(t)\right)}-\frac{F\left(r_{i}^{-1}(t)\right)}{p_{i}\left(r_{i}^{-1}(t)\right)}, & \text { if } t \geq T_{1},\end{cases} \\
& B x(t)= \begin{cases}B x\left(T_{1}\right), & \text { if } t \in\left[T_{0}, T\right], \\
-\frac{1}{p_{i}\left(r_{i}^{-1}(t)\right)} \int_{r_{i}^{-1}(t)}^{\infty} q(s) G(x(g(s))) \mathrm{d} s & \\
+\frac{1}{p_{i}\left(r_{i}^{-1}(t)\right)} \int_{r_{i}^{-1}(t)}^{\infty} u(s) H(x(h(s))) \mathrm{d} s, & \text { if } t \geq T_{1} .\end{cases}
\end{aligned}
$$


The function $r^{-1}$ used in the definition of the operators $A$ and $B$ is the inverse function of $r(t)$, which exists if $r(t)$ is assumed to be monotonic. Further more, note that $r^{-1}(r(t))=t$.

Remark 3. For the results in this section, we assume none of the conditions (4), $G$ is nondecreasing and $x G(x)>0$ unlike the corresponding results in $[3,4]$.

Taking into consideration of all the results i.e., Theorems $1-3,6$, and 8 and Remark 2, we obtain the following theorem.

Theorem 9. Suppose that (2)-(4), (6), and (7) hold. Assume $p_{j}(t)$ in (1) to satisfy one of the four conditions (15), (30), (34), and (53). Then, (5) is both necessary and sufficient condition for every solution of (1) to be oscillatory or tending to zero as $t \longrightarrow \infty$.

Remark 4. If $p_{i}(t)=p(t)$ and $p_{1}(t)=p_{2}(t)=\cdots=$ $p_{i-1}(t)=p_{i+1}(t)=\cdots=p_{k}(t)=0$, then conditions (15), (30), (34), and (53) due to the boundedness of $p_{j}(t)$ reduce to the following conditions (i) $0 \leq p(t) \leq p<1$, (ii) $-1<-p \leq p(t) \leq 0$, (iii) $-p_{1} \leq p(t) \leq-p<-1$, and (iv) $p_{1} \geq p(t) \geq p>1$, respectively. These conditions are assumed in $[1,3-5]$.

Remark 5. Taking the Remarks 1, 3, and 4 into consideration, Theorem 9 generalizes and improves the results in $[3,4]$.

\section{Application to Neutral Equations with Oscillating Coefficients}

In this section, we find sufficient conditions so that every solution of the first-order neutral differential equation (11) oscillates or tends to zero as $t \longrightarrow \infty$, where $v(t)$ is allowed to change sign. Let $v^{+}(t)=\max \{v(t), 0\}$ and $v^{-}(t)=$ $\max \{-v(t), 0\}$. Then, $v(t)=v^{+}(t)-v^{-}(t)$, and equation (11) can be written as

$$
\begin{aligned}
& \left(y(t)-\sum_{j=1}^{k} p_{j}(t) y\left(r_{j}(t)\right)\right)^{\prime}+v^{+}(t) G(y(g(t))) \\
& -v^{-}(t) G(y(g(t)))=f(t) .
\end{aligned}
$$

Now, we proceed as in the previous section by setting $q(t)=v^{+}(t), u(t)=v^{-}(t)$, and $H(x)=G(x)$. Assumptions (5)-(7) would become

$$
\begin{aligned}
& \int_{t_{0}}^{\infty} v^{+}(t) \mathrm{d} t=\infty, \\
& \int_{t_{0}}^{\infty} v^{-}(t) \mathrm{d} t=\infty,
\end{aligned}
$$

$G$ is bounded,

respectively.

Therefore, the study of (11) reduces to the study of (1) in results Theorems $1-3,6,8,9$, and Remark 2. From Theorem
9 , we have the following theorem for (11) where $v(t)$ changes sign.

Theorem 10. Suppose that (2)-(4), (77), and (78) hold. Assume $p_{j}(t)$ in (11) to satisfy one of the four conditions (15), (30), (34), or (53). Then, (76) is both necessary and sufficient condition for every solution of (11) (with $v(t)$ changing sign) to be oscillatory or tending to zero as $t \longrightarrow \infty$.

From Theorems 4 and 5, we have the following results for NDDE (11) with $v(t)$ changing sign.

Theorem 11. Suppose that (2), (3), (77), and (78) hold. Assume that (38) and (39) hold, then every solution of (11) (with $v(t)$ changing sign) oscillates.

Theorem 12. Assume conditions (2)-(4), (77), (78), and (38) to hold. Further, assume $g\left(r_{i}(t)\right)=r_{i}(g(t))$ for $i=1,2, \ldots, k$. Let there exist a real $\mu>0$ such that for $x_{i}>0, i=1,2, \ldots, k+1$, and $u>0$, (44) and (45) hold. Suppose that (46) holds where $q(t)=v^{+}(t)$. Further, suppose that there exists a real $\alpha>0$ such that $r_{i}^{\prime}(t) \geq 1 / \alpha$. Then, every solution of (11) (with $v(t)$ changing sign) oscillates or tends to zero as $t \longrightarrow \infty$.

For the results in this section, we need G to be bounded, continuous, and to satisfy (2). The prototype of such a function $G(y)$ is $y 2 n \operatorname{sgn}(y) /(1+y 2 n)$. The following examples illustrate Theorem 10 .

Example 3. Consider the higher-order NDDE:

$$
\begin{aligned}
& \left(y(t)-(1 / 2 e) y(t-1)-\left(1 / 2 e^{2}\right) y(t-2)\right)^{\prime} \\
& \quad+q(t) y(t-3)=f(t),
\end{aligned}
$$

where

$$
\begin{aligned}
& q(t)=\left\{\begin{array}{l}
\sin (t), \quad 2 k \pi \leq t \leq(2 k+1) \pi, \quad k=0,1,2, \ldots \\
\frac{\sin (t)}{t^{4}}, \quad(2 k+1) \pi \leq t \leq(2 k+2) \pi, \quad k=0,1,2, \ldots,
\end{array}\right. \\
& f(t)= \begin{cases}\sin (t) e^{-t+3}, \quad 2 k \pi \leq t \leq(2 k+1) \pi, \quad k=0,1,2, \ldots, \\
\frac{\sin (t) e^{-t+3}}{t^{4}}, \quad(2 k+1) \pi \leq t \leq(2 k+2) \pi, \quad k=0,1,2, \ldots .\end{cases}
\end{aligned}
$$

Clearly,

$q^{+}(t)= \begin{cases}\sin (t), & 2 k \pi \leq t \leq(2 k+1) \pi, \quad k=0,1,2, \ldots \\ 0, & (2 k+1) \pi \leq t \leq(2 k+2) \pi, \quad k=0,1,2, \ldots,\end{cases}$

$q^{-}(t)= \begin{cases}0, & 2 k \pi \leq t \leq(2 k+1) \pi, \quad k=0,1,2, \ldots \\ \frac{\sin (t)}{t^{4}}, & (2 k+1) \pi \leq t \leq(2 k+2) \pi, \quad k=0,1,2, \ldots\end{cases}$ 
It may be verified that the NDDE (79) satisfies all the conditions of Theorem 10. Hence, every solution of (79) oscillates or tends to zero as $t \longrightarrow \infty$. As such, it admits a positive solution $y(t)=e^{-t}$ which tends to zero as $t \longrightarrow \infty$.

Example 4. Consider the following higher-order NDDE:

$$
\begin{aligned}
& \left(y(t)-(1 / 2 e) y(t-1)-\left(1 / 2 e^{2}\right) y(t-2)\right)^{\prime} \\
& \quad+q(t) G(y(t-3))=f(t),
\end{aligned}
$$

where $G(u)=u^{2} \operatorname{sgn}(u) /\left(1+u^{2}\right)$ and $q(t)$ as in (80) and

$$
f(t)= \begin{cases}e^{6}\left(e^{2 t}+e^{6}\right)^{-1} \sin (t), & 2 k \pi \leq t \leq(2 k+1) \pi, k=0,1,2, \ldots \\ \frac{e^{6} \sin (t)}{\left(e^{2 t}+e^{6}\right) t^{4}}, & (2 k+1) \pi \leq t \leq(2 k+2) \pi, \quad k=0,1,2, \ldots,\end{cases}
$$

satisfies all the conditions of Theorem 10. Hence, every solution of (84) oscillates or tends to zero as $t \longrightarrow \infty$. As such, it admits a positive solution $y(t)=e^{-t}$ which tends to zero as $t \longrightarrow \infty$.

Remark 6. The results of this article seem to be significant as no result in literature can be applied to the NDDEs (79) and (84).

\section{Conclusion}

The open problem [1] (Problem 2.8.3, p. 57) says the following:

Extend the following result to equations with oscillating coefficients.

Theorem 2.3.1 in [1]: under the assumptions that $v(t) \geq 0$ and

$$
\liminf _{t \longrightarrow \infty} \int_{t-\tau}^{t} v(s) \mathrm{d} s>e^{-1},
$$

every solution of

$$
y^{\prime}(t)+v(t) y(t-\tau)=0, \quad t \geq t_{0}
$$

oscillates.

If we put $f(t)=0, G(y)=y$, and $k=0$ in (11), then the following corollary follows from Theorem 10.

Corollary 1. Suppose that (76) and (77) hold. Then, every bounded solution of

$$
y^{\prime}(t)+v(t) y(g(t))=0
$$

(with $v(t)$ changing sign) oscillates or tends to zero as $t \longrightarrow \infty$.

Again, if we put $f(t)=0, k=0$ in (11), then the following corollary follows from Theorem 10.

Corollary 2. Suppose that (2), (76), and (77) hold. Then, every solution of

$$
y^{\prime}(t)+v(t) G(y(g(t)))=0
$$

(with $v(t)$ changing sign) oscillates or tends to zero as $t \longrightarrow \infty$. Or, equivalently every unbounded solution of (89) oscillates.

Note that (86) implies

$$
\int_{t_{0}}^{\infty} v(t) \mathrm{d} t=\infty
$$

Further, (76) is equivalent to (90) under the assumption (77). Thus, Corollaries 1 and 2 answer the open problem [1] (Problem 2.8.3, p.57) partially. Further, as the condition " $G$ is nondecreasing" is not assumed, and $v(t)$ in NDDE (11) has no fixed sign; therefore, by Remark 4, Theorem 10 of this article improves and generalizes the results in [7] (Theorem 2.2, Theorem 2.4, and Theorem 2.5) because of their additional assumptions (i) $\sigma>\alpha$ or $\sigma<\alpha$, (ii) $q(t)>u(t-\sigma+\alpha$ ), and (iii) another sublinear condition on $G$, while studying the NDDE (9). Further, in this paper, Theorem 10 improves and generalizes the results in [4] (Theorems 2.2, 2.3, 2.5, 2.6, and Corollary 2.7) for the NDDE (8).

\section{Data Availability}

The data used are published research articles in different referred journals which can be assessed in their websites.

\section{Conflicts of Interest}

The authors declare that they have no conflicts of interest.

\section{References}

[1] I. Gyori and G. Ladas, Oscillation Theory of Delay-Differential Equations with Applications, Clarendon Press, Oxford, UK, 1991.

[2] P. Das and N. Misra, "A necessary and sufficient condition for the solutions of a functional differential equation to be oscillatory or tend to zero," Journal of Mathematical Analysis and Applications, vol. 205, no. 1, pp. 78-87, 1997.

[3] N. Parhi and R. N. Rath, "On oscillation criteria for a forced neutral differential equation," Bulletin, Institute of Mathematics, Academia Sinica, vol. 28, pp. 59-70, 2000.

[4] N. Parhi and R. N. Rath, "Oscillation criteria for forced first order neutral differential equations with variable coefficients," Journal of Mathematical Analysis and Applications, vol. 256, no. 2, Article ID 525541, 2001.

[5] N. Parhi and R. N. Rath, "Oscillation of solutions of a class of first order neutral differential equations," Journal of the Indian Mathematical Society, vol. 71, pp. 175-188, 2004.

[6] N. Parhi and S. Chand, "On forced first order neutral differential equations with positive and negative coefficients," Mathematica Slovaca, vol. 50, pp. 81-94, 2000.

[7] R. N. Rath and N. Misra, "Necessary and sufficient conditions for oscillatory behavior of solutions of a orced non linear neutral equation of first order with positive and negative coefficients," Mathematica Slovaca, vol. 54, pp. 255-266, 2004.

[8] R. N. Rath, P. P. Mishra, and L. N. Padhy, "On oscillation and asymptotic behavior of a neutral differential equation of first order with positive and negative coefficients," Electronic Journal of Differential Equations, vol. 2007, no. 1, pp. 1-7, 2007. 
[9] J. S. Yu and Z. Wang, "Some further results on oscillation of neutral differential equations," Bulletin of the Australian Mathematical Society, vol. 46, pp. 149-157, 1992.

[10] N. Parhi and R. N. Rath, "On oscillation and asymptotic behaviour of solutions of forced first order neutral differential equations," Proceedings Mathematical Sciences, vol. 111, no. 3, pp. 337-350, 2001.

[11] R. Rath and C. Behera, "Oscillatory and asymptotic behavior of a first order neutral equation of discrete type with variable several delay under $\Delta$ sign," International Journal of Mathematics and Mathematical Sciences, vol. 2018, Article ID 4586176, 2018.

[12] H. L. Royden, Real Analysis, MacMilan Publ. Co., New York, NY, USA, 3rd edition, 1988.

[13] L. H. Erbe, Q. K. Kong, and B. G. Zhang, Oscillation Theory for Functional Differential Equations, Marcel Dekker, New York, NY, USA, 1995.

[14] R. N. Rath, N. Misra, P. P. Misra, and L. N. Padhy, "Non oscillatory behaviour of higher order functional differential equations of neutral type," Electronic Journal of Differential Equations, vol. 2007, no. 163, pp. 1-14, 2007. 